\title{
What in-patients want: a qualitative study of what's important to mental health service users in their recovery (Wayfinder Partnership)
}

\author{
Joanna Bredski ${ }^{1}$, Kirsty Forsyth ${ }^{2}$, Debbie Mountain $^{3}$, Michele Harrison $^{4}$, Linda Irvine ${ }^{5}$ and Donald \\ Maciver $^{6}$
}

1. Consultant General Adult Psychiatrist, NHS Fife, 2. Professor, School of Health Sciences, Queen Margaret University,3. Consultant Rehabilitation Psychiatrist, NHS Lothian, 4. Lead Research Practitioner, School of Health Sciences, Queen Margaret University, 5. Strategic Programme Manager, Mental Health and Wellbeing, NHS Lothian 6. Reader, School of Health Sciences, Queen Margaret University,.

\begin{abstract}
Purpose: The purpose of this paper is to present a qualitative analysis of the facilitators of recovery in inpatient psychiatric rehabilitation from the service users' perspective.

Design/methodology/approach: Interviews with 31 in-patients were coded and analysed thematically at an interpretive level using an inductive approach.

Findings: The dominant themes identified were hope, agency, relationships and opportunity. Totally, 20 subthemes were identified. Agency was more important to men than women and agency, hope and relationships were all more important to detained patients.

Research limitations/implications: Interview data were collected in writing rather than taped. The results may not be transferrable to patient populations with significantly different demographic or service factors.

Practical implications: Services need to target interventions at the areas identified by service users as important in their recovery. The findings suggest both environmental and relational aspects of care that may optimise recovery. Services also need to be able to measure the quality of the care they provide. A brief, culturally valid and psychometrically assessed instrument for measuring the recovery orientation of services is required.

Originality/value: As far as the authors are aware no qualitative work to date has examined the recovery experiences of psychiatric in-patient service users in order to understand what services require to do to enable recovery from their perspective. The conceptual framework identified in this paper can be used to develop a service user self-report measure of the recovery orientation of services.
\end{abstract}

\section{Introduction}

Anthony's popular definition of recovery is of "a deeply personal, unique process of changing one's attitudes, values, feelings, goals, skills and/or roles. It is a way of living a satisfying, hopeful, and contributing life even with limitations caused by the illness" (Anthony, 1993, p.527). Interestingly, recovery has also been defined as the lived experience of rehabilitation (Deegan, 1998). Psychiatric rehabilitation offers "a whole systems approach to recovery from mental illness that maximizes an individual's quality of life and social inclusion by encouraging their skills, promoting independence and autonomy in order to give them hope for the future and leads to successful community living through appropriate support", (Killaspy et al. 2005, p.163). The World Health Organization (WHO) priorities for mental health services focus strongly on recovery principles (WHO, 2005). The WHO also recognises the importance of service user experience as a basis for designing mental health services (WHO, 2005). The authors are not aware of any published research to date that has aimed to define what it is that service users want from in-patient psychiatric rehabilitation in order to facilitate their own recovery. In a narrative synthesis of the literature on personal recovery, Leamy et al., (2011), identified five 'recovery process' themes; 'connectedness' (predominantly describing relationships), 'hope and optimism about the future', 'identity', 'empowerment' (similar to agency) and 'meaning in life', which includes meaningful social roles. However, there is limited published work on how service users evaluate the recovery process within hospital and community based mental health services and the recovery orientation of services (O'Connell et al., 2005). There are studies which have made recommendations for how to incorporate recovery indicators into mental health systems (Onken et al., 2002). However, Donnelly et al., (2011) failed to recommend the use of any of the service user rated recovery orientated measures they reviewed.

The Wayfinder Partnership is a Knowledge Transfer Partnership between Queen Margaret University and NHS Lothian. The aim of the Partnership is to redesign the psychiatric rehabilitation pathway in Edinburgh using both the evidence base and stakeholder views. This paper presents the work of the Wayfinder: Have Your Say project, which is part of the larger Wayfinder Partnership. 
The aims of the Wayfinder: Have Your Say project were:

1. To identify, using qualitative research methods, the facilitators of recovery in in-patient psychiatric rehabilitation from the service users' perspective in order to inform the rehabilitation pathway redesign;

2. To develop a conceptual framework from which a service user self-report outcome measure of the recovery orientation of services can be built.

The qualitative analysis is presented in this paper. The pilot and psychometric evaluation of the outcome measure that has been developed will be presented in a future article.

\section{Method}

This cross-sectional study used semi-structured interviews. Anthony's (1993) definition of recovery was read to the participants. Participants were then asked the following questions:

1. what is important in your recovery?

2. why is this important?

3. what about the service you receive has been helpful?

4. what needs to be changed?

Answers were probed and participants asked to expand on important points. The 4 questions were designed following consultation with an expert reference group consisting of service user advocates, qualitative researchers and senior mental health clinicians. Data gathering forms were used to record the data. The above questions were printed on the data gathering form and underneath a blank space left to allow answers to be recorded. Participants were interviewed individually by service user advocates working in pairs. The service user advocates were members of the Royal Edinburgh Hospital Patients' Council who provide a collective advocacy service to the in-patient units. They received training from J.B. on conducting the interviews. The service user advocates were known to many of the participants because of their role in providing collective advocacy. The service user advocates were chosen to collect the data because of their unique position of having a role in advocating for service users, being already known to many of the service users and not being a part of the clinical care team. When not known to the service user their status was disclosed. It was thought that this would allow participants the best opportunity to answer as openly as possible without concerns about the impact this may have on the care they receive.

The interviews were carried out between $1^{\text {st }}$ April and $31^{\text {st }}$ May 2011 and were recorded live in writing during the interview with as much as possible recorded verbatim by one of the service user advocates in each pair. A further discussion regarding this collection method in included in the Limitations section. The majority of interviews were carried out in private rooms within the in-patient units. For service users who had concerns about using this as the location interviews were conducted in an activity centre and coffee bar within the hospital site. The interviews varied in length and participants were given as much time as they wished to give their responses.

\section{Participants}

Our inclusion criteria were all in-patients in Rehabilitation Service wards at the Royal Edinburgh Hospital during the period of data collection. There were no exclusion criteria. At the time of data collection the in-patient Rehabilitation Service consisted of four wards. Two mixed wards with 25 beds and 15 beds respectively offered high-dependency rehabilitation to those who were highly symptomatic and had significant risk histories, as well as challenging behaviours. The two other wards provided longer-term complex care offering longer term admission - often for several years - to those with a high level of disability from complex co-morbid conditions with associated significant risks to themselves or others. One was a 19 bedded male only ward and the other a 15 bedded ward where the need for on-going monitoring and treatment of serious physical health problems is high. A full typology of in-patient rehabilitation services has been provided elsewhere (Royal College of Psychiatrists, 2009).

\section{Ethics}

The South East Scotland Research Ethics Service confirmed that ethical approval was not required under NHS research governance arrangements. The project was considered a service evaluation focussed on service user's views on the impact of the service on their recovery in one locality. Rigorous ethical standards were adhered to regarding consent, capacity and potential for coercion. 
Participants were recruited in three ways: advertisements were placed on notice boards in the in-patient unit, rehabilitation staff asked service users in routine appointments if they would be interested in participating and visits to the unit were scheduled by Patients' Council advocates who asked service users if they would like to participate. Detailed information about what participation would involve was scripted by J.B. and provided by the interviewers. Participants were also provided with written information including a telephone number to contact if they had any questions or concerns. Consent was received by the interviewers prior to completing the interview. Service users who were viewed as unable to consent were identified by their Consultant Psychiatrist or Responsible Medical Officer and were not invited to participate in the study.

\section{Analysis}

The data set was analysed thematically using an inductive approach: i.e., the themes were identified 'bottom up' and are strongly linked to the data themselves, rather than 'top down' and driven by theory (Patton,1990). An interpretive level of analysis was used: this aims to identify underlying ideas, assumptions and conceptualisations that are theorised as informing the semantic content of the data (Braun and Clarke,2006; Boyatzis,1998). Patterns in the data were coded by one rater (J.B.). The appropriateness of the coding frame was checked through progressive iterations and reapplied to earlier transcripts as it developed. Nvivo 8 (2008, QSR International Pty Ltd, www.qsrinternational.com) on Microsoft Windows was used for indexing the material. Data that did not seem to fit into the coding frame were actively sought. Codes with similar information were merged, irrelevant codes were pruned and an initial identification of themes was conducted. The codes were analysed and combined to form overarching themes with subthemes. The themes were reviewed and refined until they formed a coherent pattern. The data set was then re-coded to ensure that the themes accurately represented the meanings in the data set as a whole. The themes were further refined and the data in them analysed to ensure that the scope of each theme could be clearly defined and had accompanying illustrative quotations. A final consultation was held with senior mental health clinicians, qualitative researchers and service user advocates, after which the framework was further refined. The prevalence of each theme was counted as the number of cases in which the theme occurred. Microsoft Excel was used to compare groups on the basis of age, gender and the use of compulsory measures.

\section{Results}

Thirty one service users participated. Twenty were male and eleven female. Eighteen were admitted for high dependency rehabilitation and thirteen for complex care rehabilitation. Thirty were white British and one Black and minority ethnic. The mean age of the service users was 43 (range 19-70, S.D. = 14.4). Unit staff were asked to record the participant's diagnosis, date of transfer to the service and whether or not the person was detained under the Mental Health (Care and Treatment) (Scotland) Act 2003 (MHA). The following diagnoses were identified: schizophrenia $(n=19)$, schizoaffective disorder $(n=3)$, personality disorder $(n=2)$, bipolar disorder $(n=1)$ and six service users did not wish their diagnosis to be recorded. Eighteen service users were detained under the MHA, six were not and seven did not wish this to be recorded.

The interviews provided rich descriptions of the facilitators of recovery as perceived by service users.

Four themes that promote recovery were identified: relationships, hope, agency and opportunity. Twenty subthemes were identified.

\section{Relationships}

Family and friends

Having enough access to family and friends and receiving support from staff with these relationships if required was perceived as highly important to individuals in their recovery. Relationships with family and friends were seen as beneficial because they increased a person's support network and offered greater access to local communities and a sense of social inclusion. They may also promote a more positive sense of identity and offer welcome respite from the in-patient environment for some;

'Talking to friends and family about everyday issues and not hospital talk' (P14). 
These relationships also helped to alleviate boredom and were linked with feeling more hopeful. Some individuals acknowledged the role of services in helping them to manage their relationships with friends and family members.

'Service has helped with my family relationships' (P21).

However, in some cases services were seen to be the cause of reduced contact with family members: either because of ward environments that were considered to be hostile to visitors or because of a perceived lack of independent leisure time away from the ward.

'Mum and dad no longer visit because of atmosphere in ward' (P25).

Staff

Participants discussed several features of their relationships with staff which they viewed as promoting recovery. Support from staff was identified as enabling people in terms of their functioning and this in turn promoted a more positive sense of identity. One participant (P20) described that the

'Nurses helped me to get out when it was difficult for me... increasing my confidence'.

Another described how

[there's a] supported environment on rehab wards as opposed to other wards I have been on.' (P14)

Hence, many participants viewed themselves as receiving the right amount of support for them:

"the help that I need is usually pretty much when I need it' (P5).

However, a small minority of participants viewed that they received more support than they required. Self confidence and self esteem were reported to be promoted by positive relationships with staff and positive ward environments in which service users were treated with respect and as individuals:

'Being talked to as a person not a "type" of illness' (P24).

The experience of being listened to and cared for was linked to promoting self-esteem. The experience of relationships with ward based nursing staff was the most commonly described. This is perhaps unsurprising given the frequency and regularity of contact with this group of staff and the depth of relationships that can develop e.g. with key workers over time. A smaller number of people reported that their psychiatrist listening to them and caring for them was also very important.

Many participants perceived that they were listened to and cared for by staff, sometimes quite remarkably despite overwhelmingly negative views about other parts of the service:

...some nurses care and do listen to me' (P4).

However, a small minority viewed that they were not treated with care by staff. Participant 5 described that at times it "feels like I'm treated like a child", and experiencing "punitive threats from staff to comply with ward routine".

\section{Agency}

Human agency is the capacity for a person to make a choice and to enact it on the world (von Peter, 2013). We have used the term here to describe purposeful, goal directed activity for which an agent implements direct control over their own behavior. We separated this from the theme of opportunity below, which we've used to describe what the environment provides for the individual, which is largely determined rather than influenced directly by the agency of the individual.

\section{Choices}


Some service users viewed themselves as not being supported to make choices and for most people this involved choices about the services they receive. For service users who are detained it is of course the case that a number or important decisions about care and treatment will not be as a result of the individual's choice. A small number found services over-protective - 'too helpful ' (P28)- and reported that this undermined their ability to make choices for themselves.

\section{Control of problems}

One service user described a sense of empowerment around managing her mental health problems, and planned:

'To keep working at my symptoms [and] psychological health - I look after me.' (P22).

As above, some participants experienced services as over-protective. One person described a wish for:

'Some responsibility...care and treatment from staff only when I need it' (P21).

A minority of patients viewed that they did not need support. Most, however, valued support, but wished to have more control over their problems whilst still being able to access the support they required.

\section{Information ownership}

It was important to a number of service users to feel that they had ownership of a good understanding of their mental health, treatment and progress. Having medication and diagnoses explained was desirable to some, but in particular receiving regular feedback from staff on the progress of their recovery was thought to be empowering. Service users also wanted information about what to expect in future particularly in relation to community living:

'Having clarity on stages of the road [and] advice on support services' (P 30).

A minority of participants did not feel that they received regular feedback on their recovery and the potential for them moving to the community. This was perceived as a cause for loss of hope.

\section{Opportunity}

Opportunity refers to a favourable combination of circumstances: in this context it describes the environmental resources e.g. employment that are available to a service user that promote their recovery. Service users make a distinction between the 'on' and 'off' ward environments with access to opportunities out with the ward environment being more desirable particularly to those who viewed that they did not require the level of support or restriction they were subject to. Some of the subthemes relate more strongly to one environment than the other, but a number are continuous across the two environments.

Meaningful activities

Taking part in a wide range of activities including normal activities of daily living, occupational therapy groups, art work, exercise and education was very important to people. Activities were viewed as promoting happiness, alleviating boredom and increasing people's opportunities further by affording them access to both the hospital community and the wider community. Being able to take part in meaningful activity was associated with a sense of hopefulness. It was also viewed as helpful in managing symptoms of illness:

'An education, always being busy...it focusses my mind doing things which keep me away from isolation with visions.' (P6)

I am glad that I can go to voluntary work three to four times every week...

because it's like all the things I would expect to describe in a "well" person's life.' ( P5)

Involvement in activity was perceived as positive even in those who viewed staff and services as on the whole unhelpful:

'No one has been helpful...education class has been helpful.' (P3)

Work

Although work is a form of meaningful activity, it was of high importance to a large number of service users and as such a separate subtheme was created. Some participants perceived their voluntary work as one of the most important facilitators in their recovery: 
'Volunteering...doing things outside [of the] ward...feeling useful.' (P11)

Voluntary work was linked with giving an individual a sense of self-worth and also promoting hopefulness. As most voluntary work takes place outside of the ward environment it may also offer further opportunities such as inclusion in communities.

Meaningful routine

Meaningful routine refers to the structure of activities including support that a service user has access to and is continuous between the 'on' and 'off' ward environments. Views were mixed about whether the service promoted meaningful routine, but there was a strong consensus view that this was necessary. Having a meaningful routine was perceived as promoting hopefulness and reducing boredom. Daily routines were also viewed by service users as giving them a sense of stability, which may in turn promote a sense of security and safety.

Boredom

Although boredom may be viewed as the absence of meaningful activity, routines and work, service users often viewed alleviating boredom as an end in itself. Many participants perceived boredom as a significant problem to them in their recovery. Having access to friends and family was seen as helpful in reducing boredom and not feeling bored was felt to promote hopefulness.

Independent leisure time off ward

Having enough time away from the ward alone or with friends and family was perceived as important in recovery by a large number of service users. Those who did not have enough opportunities to have time away from the ward reported a sense of being 'trapped' and of wishing for 'more freedom'. (P8) A number of service users viewed leisure time away from the ward as giving them hope as well as reducing boredom and supporting them in their relationships. Some service users were frustrated by and unable to understand the need for restriction. The experience of restriction that was felt to be unnecessary was perceived as undermining of agency and self-worth.

Peaceful environment

Having a calm ward environment was important to service users:

'Makes me feel peaceful when [the] ward feels very homely.' (P11)

Some service users worried about areas of poor repair such as dampness, missing furniture, ageing decorations and soft furnishings, and suggested improvements. It would appear that for some service users at least an environment would not be perceived as peaceful unless it was well maintained.

Safe and secure environment

A large number of participants viewed feeling safe on the ward as highly important. Only one reported not feeling safe. Some had clearly had experiences of feeling highly at risk in the community:

'Sense of safety compared to bullying by children when I was out of hospital.' (P25)

'Safe and secure environment...staying on the ward long term has helped as I was in and out on acute wards.' (P22)

The feeling of safety was influenced by the stability and predictability of the ward environment rather than by security measures such as doors being locked.

Privacy

Privacy for most people meant having their own room. Most people who reported that privacy was important in their recovery did not have their own room.

\section{Hope}

Hopefulness

A sense of hope was important and a mixture of hopefulness and hopelessness was expressed by participants. A long stay in hospital was perceived as causing hopelessness particularly in patients who were detained and those who felt that they did not need to be in hospital.

Future focus

Looking forward rather than back was perceived as helpful:

'Concentrating on the good things in the future'.

In most cases this involved a wish to focus on moving out of hospital and into to the community. One 
participant (P18) expressed being able to see the 'light at the end of the tunnel'. Individuals viewed receiving information from staff about their progress towards community living and having support that actively prepared them for this beneficial.

'I feel quite hopeful because I am seeing the housing officer soon'. (P8).

Other

Three strong subthemes did not fit into the four identified overarching themes described above. These themes related to service users views about their mental health and their treatment specifically in terms of medication and finally their ability to manage their own money.

Understanding of mental health problems

A small number of participants reported that having an understanding of their mental health problems was important in their recovery:

\section{'Acknowledging that I am not well.' (P22)}

More commonly service users reported a lack of understanding of the need for their hospital admission and/or need for support. Those who perceived that they did not require hospital services tended to view staff as either unhelpful or too helpful. This perspective was associated with hopelessness and frustration. It was more likely for service users with this perspective to report difficulties in their relationships with staff and an insufficient sense of control and ability to make choices.

\section{Medication}

Interestingly, medication was very commonly reported to be important to service users in their recovery and only a small minority viewed medication as unhelpful. Most viewed medication as beneficial over all and this view was shared by most detained patients:

'It's important to the patient for the doctors to get the medicine right to give patients a good quality of life.' (P2)

Those who viewed medication as unhelpful were more likely to also report difficulty understanding their need to be in hospital or to receive the support or restrictions they were subject to.

\section{Managing money}

A small number of service users viewed having control of their finances as one of the most important facilitators of recovery. For one service user, being able to manage her own money was a part of preparing for her future:

'Money is also important, not just for cigarettes or juice, but to save up for a holiday' (P5).

Those who wished for greater financial control also tended to wish for greater access to independent leisure time away from the ward. This suggests that managing money may be of particular importance to those who are subject to more environmental restrictions.

\section{Between group differences}

We examined differences in the prevalence of each theme and subtheme by sex, age and whether or not the participants were detained. The prevalence of each theme was counted as the number of cases in which the theme occurred.

\section{Sex}

The theme of agency was over twice as prevalent in the reports of men. The subtheme of self-worth was almost twice as common in women's reports. The themes of hope, opportunity and relationships were broadly similar in prevalence between the genders. Men had a preference for staff to support and help and women a preference for staff to listen and care. A peaceful ward environment was more important to women and meaningful routine more important to men.

Detention status

The themes of hope and agency were reported by detained patients when compared with non 
detained patients with over twice the prevalence. Perhaps more interestingly, the prevalence of the theme of relationships was almost four times higher in detained patients. In detained patients the subthemes of privacy and independent leisure time away from the ward were two and three times more prevalent respectively. The theme of opportunity was similar in prevalence in the two groups.

Age

The theme of relationships was the most prevalent theme in all age groups other than in those aged 55 or over. Interestingly, no-one over the age of $55(n=7)$ reported relationships with staff as being important in their recovery. The subtheme of future focus was also absent in those aged over 55. However, the subtheme of meaningful activity was more prevalent in this age group than others.

\section{Discussion}

We aimed to explore the views of psychiatric in-patients about what's important to them in their recovery. The analysis revealed valuable insights into what services can do to promote the recovery of service users. Participants regarded their recovery as facilitated by hope; a sense of agency; having good relationships with staff, friends and family and having access to opportunities that are meaningful to them. All of these were perceived as being promoted by ward environments that offer privacy, safety and peacefulness as well as staff who listen, care, support, help and treat patients with respect and as individuals.

The 'recovery processes' identified in Leamy et al.'s (2011) work has much in common with the themes of our analysis. However, the concept of personal recovery does not capture the importance of environmental factors such as access to privacy on a unit that feels safe and secure (Onken et al.,2007). It is recommended that services focus on promoting recovery in an environment that optimally facilitates personal recovery both within and out with the in-patient unit including providing opportunities such as access to employment. The concept of opportunity has been highlighted elsewhere as a fundamental part of the recovery process and the importance of being a valued and contributing member of a community with access to opportunities offered by the community has been emphasised (South London and Maudsley NHS Foundation Trust and South West London and St George's Mental Health NHS Trust 2010). We do not wish to co-opt the concept of personal recovery - that began after all as a service user movement - into describing a model of care provided by mental health services. Instead we view that our conceptual model of recovery, which does include environmental factors, has clinical utility in the context in which it has been designed to be used - to develop (and assess) the ability of services to facilitate recovery for their service users.

It is worth noting that the theme of identity was not identified in our analysis. Identity is widely held to be an important part of personal recovery (Leamy et al., 2011), a view that is shared by the authors. The concept of identity is however an abstract one and certainly less easy to define than for example having a job or not feeling bored. It may be that identity is less important to in-patient service users or perhaps less immediately pertinent. It may also be that the language to describe such an abstract concept is not available to some in-patient service users or that identity is not a concept discussed with service users by staff as readily as e.g. hope.

\section{Recovery focused services}

Over fifty member countries have adopted the WHO action plan for mental health services in which recovery principles are strongly represented. WHO priorities include the design and implementation of services that promote 'rehabilitation, care and recovery' (WHO, 2005). Also prioritised is the recognition of the experience of service users as an important basis for planning and developing services (WHO, 2005).

To the best of our knowledge, our study is the first qualitative study to have investigated the views of psychiatric rehabilitation in-patient service users on what's important in their recovery. At the level of care provision for an individual service user the themes and subthemes we identified may be used as an aide memoire when goal planning. As not all of the themes will be meaningful for each service user, however, a one-size-fits-all approach would be unlikely to promote recovery. At a service level, however, all of the themes and subthemes could be considered when aiming to provide an optimal service to facilitate recovery. They could be used to focus the use of specific interventions - such as psycho-education to try to improve service users' understanding of their mental health or supported work programmes - with the aim of promoting the functional, personal and social recovery of those 
using services (Farkes et al., 2007; Lloyd et al., 2008).

The between group differences may also be borne in mind when planning at an individual and a service level. Detained patients not only appear to prioritise agency - denied them at least partially by compulsory treatment - but also hope and particularly relationships with staff. Older adults appear to prioritise meaningful activity over relationships. Agency appears to be of particular importance to men. For example, in a male only unit in which the majority of service users are detained it may be particularly important to try to support service users to make their own meaningful choices, retain a sense of control over their problems and to manage their own money when possible. Decisions about a service user's access to money and time away from the ward are often taken by staff with minimisation of risk being the key priority. This must be balanced against the impact that restrictions including the ability to manage money may have on a service user's sense of agency as well as their ability to achieve positive relationships with staff, both of which are central to recovery.

Our findings could also be used to guide certain aspects of the design of ward environments - which optimally would feel secure and peaceful with single rooms for privacy - and ward programmes, which are structured, dependable and meaningful. Relationships with staff must be caring, supportive, helpful and respectful and staff should endeavour to focus on the future and prepare service users with the information that they wish for in relation to their mental health, treatment and progress.

\section{Limitations}

The qualitative design allowed us to obtain an in depth understanding of service users' views about their recovery. The sample contains service users who consented to participate in the study. Other perspectives might be held by those who were less inclined to share their experiences. The coding was carried out by one rater and as such we were unable to measure the consistency of coding between two or more raters. However, the coding was discussed with and refined by qualitative researchers throughout the process of analysis.

The between group differences in the prevalence of themes are interesting, but require to be viewed with caution. The numbers are small and as the study was not designed to yield quantitative data no attempt has been made to examine for statistical significance.

The interview data were recorded live by note taking in writing by service user advocates rather than recorded by tape. This collection method was chosen because the service user advocates viewed that the use of tape recording would deter service users from participating. The service user advocates were asked to record the responses of participants - as much as possible - verbatim. This method of data collection, however, does place limitations on the data collected. There is potential bias if the service user advocates position and role influenced how participants responded and how comments were recorded, and importantly on the ability of the researchers to verify it. The researchers did not collect the data themselves and could not listen to recordings and this may lead to detachment from the narrative given by the service user. Several meetings took place between the lead researcher and the service user advocates to discuss the data collected in an attempt for the researchers to gain as much understanding as possible of the service users' narratives within the above limitations.

There are no nationally agreed service specifications for psychiatric rehabilitation and rehabilitation services across the country vary in ways that are not obviously related to need (Killaspy et al., 2005; Mountain et al., 2009). The Faculty of Rehabilitation and Social Psychiatry of the Royal College of Psychiatry has recommended a model for comprehensive rehabilitation services (Royal College of Psychiatrists, 2009). This model includes high dependency and complex care rehabilitation as well as community and secure rehabilitation and highly specialist services. Our findings are from people using high dependency and complex care services only. In qualitative research, transferability refers to the extent to which the findings of a study can be applied or transferred to areas outside that which was studied (Miles and Huberman, 1994). Many people journey through different rehabilitation services during their recovery and many of those using high dependency and complex care services now may have used secure rehabilitation in the past and may use community based rehabilitation in the future. As such we view that our findings are likely to be transferrable across these four types of rehabilitation. Our findings may not be transferrable to highly specialist services such as those for autism spectrum disorder and acquired brain injury. The very low prevalence of Black and minority ethnic (BME) participants in our sample may limit transferability to areas with different prevalence 
rates. Although very few studies have looked at the experience of recovery in individuals from BME backgrounds, important differences were noted in a recent systematic review and narrative synthesis (Onken et al.,2007).

\section{Clinical implications}

It is recommended that services focus on promoting recovery in the areas that are identified by service users as important and meaningful to them. The themes and subthemes identified in this paper may be used to develop services that provide both the environmental factors and relationships with staff that service users require to optimise their experience of recovery.

This study identifies the facilitators of recovery from the perspective of people using psychiatric inpatient rehabilitation services. The recovery themes identified were incorporated with other evidence into the revised rehabilitation pathway designed as part of the Wayfinder Partnership. This pathway is currently being piloted within the service. A brief service user self-report measure of the recovery orientation of such services is required. The conceptual framework provides a basis from which a standardised outcome measure of the recovery orientation of services can be developed. The development and psychometric testing of this outcome measure will be presented in a future article.

\section{References}

Anthony, W. (1993), "Recovery from mental illness: the guiding vision of the mental health service system in the 1990s", Psychosocial Rehabilitation Journal, Vol. 16 No. 4, pp.11-23.

Boyatzis, RE. (1998), Transforming qualitative information: Thematic analysis and code development. Sage Publications, Thousand Oaks, CA.

Braun, V. and Clarke, V. (2006), "Using thematic analysis in psychology", Qualitative Research in Psychology, Vol.3 No. 2, pp.77-101.

Deegan, P.E. (1998), "Recovery: The lived experience of rehabilitation", Psychosocial Rehabilitation Journal, Vol.9 No. 4, pp.11-19.

Donnelly, M., Scott, D., McGilloway, S., O'Neill, T., Williams, J., Slade, M. (2011). "Patient outcomes: What are the best methods for measuring recovery from mental illness and capturing feedback from patients in order to inform service improvement? A report commissioned by the Bamford Implementation Rapid Review Scheme. Reference: COM 4409/10, available at http://www.publichealth.hscni.net/sites/default/files/Patient\%200utcomes.pdf (accessed on $11^{\text {th }}$ June 2014)

Faculty of Rehabilitation and Social Psychiatry of the Royal College of Psychiatrists (2009), "Enabling Recovery for People with Complex Mental Health Needs: A Template for Rehabilitation Services", available at :http://www.rcpsych.ac.uk/pdf/fr_rs_1_forwebsite.pdf (accessed on 30 January 2014).

Farkes, M., Gagne, C., Anthony, W. and Chamberlin, J. (2005), "Implementing Recovery Oriented Evidence Based Programs: Identifying the Critical Dimensions", Community Mental Health Journal, Vol. 41 No. 2, pp.141-58.

Leamy M, Bird V, Le Boutillier C, Williams J, Slade M. (2011). "A conceptual framework for personal recovery in mental health: systematic review and narrative synthesis", British Journal of Psychiatry, Vol. 199 No. 6, pp.445-452.

Killaspy, H., Harden, C., Holloway, F. and King, M. (2005), "What do mental health rehabilitation services do and what are they for? A national survey in England", Journal of Mental Health, Vol.14 No 2, pp.157-65.

Lloyd, C., Waghorn, G. and Williams, PI. (2008), "Conceptualising recovery in mental health rehabilitation", British Journal of Occupational Therapy, Vol.71 No. 8, pp.321-8.

Miles, MB. and Huberman, AM. (1994), Qualitative data analysis: An expanded sourcebook, $2^{\text {nd }}$ ed. 
Sage Publication, Thousand Oaks, CA.

Mountain, D., Killaspy, H. and Holloway, F. (2009), "Mental health rehabilitation services in the UK in 2007", Psychiatric Bullettin, Vol. 33, pp.215-8.

O'Connell, M., Tondora, J., Croog, G., Evans, A., Davidson, L. (2005). "From rhetoric to routine: Assessing perceptions of recovery orientated practices in a state mental health and addiction system", Psychiatric Rehabilitation Journal, Vol. 28 No.4, pp. 378-386

Onken,S.J., Dumont, J.M., Ridgway, P., Doman, D.H., Ralph, R.O. (2002). “Mental Health Recovery: What helps and what hinders? A National Research Project for the development of recovery facilitating system indicators", available at http://www.nasmhpd.org/docs/publications/archiveDocs/2002/MHSIPReport.pdf (accessed on 11th June 2014).

Onken, SJ., Craig, CM., Ridgway, P., Ralph, RO. and Cook, JA. (2007), "An analysis of the definitions and elements of recovery: a review of the literature", Psychiatric Rehabilitation Journal, Vol. 31 No. 1, pp.9-22.

Patton, M. (1990), Qualitative evaluation and research methods, $2^{\text {nd }}$ ed. Sage, Newbury Park, CA.

South London and Maudsley NHS Foundation Trust and South West London and St George's Mental Health NHS Trust (2010), "Recovery is for All. Hope, Agency and Opportunity in Psychiatry. A Position Statement by Consultant Psychiatrists.", available at: http://www.rcpsych.ac.uk/pdf/Recovery\%20is\%20for\%20all.pdf (accessed 30 January 2014).

von Peter, S. (2013). Agency, materiality and mental illness. Social Theory and Health, Vol. 11 No. 4, 317-326.

World Health Organization WHO (2005), "Mental Health Action Plan for Europe: Facing the Challenges, Building Solutions", availabe at :

http://www.euro.who.int/ data/assets/pdf file/0006/99735/edoc07.pdf (accessed 30 January 2014). 